\title{
Using exoplanet systems with highly elliptical orbits to search for star-planet interactions
}

\author{
John R. Hodgson II $^{1}$, Damian J. Christian ${ }^{1}$, Dennis Bodewits ${ }^{2}$ and \\ Suzanne Hawley ${ }^{3}$ \\ ${ }^{1}$ California State University, Northridge, Physics Department, 18111 Nordhoff St, \\ Northridge, California 91330-8268, USA \\ email: John.Hodgson.71@my.csun.edu \& Damian.Christian@csun.edu \\ ${ }^{2}$ Department of Astronomy, University of Maryland, College Park, \\ MD 20742, USA \\ email: dennis@astro.umd.edu \\ ${ }^{3}$ Astronomy Department University of Washington, Box 351580, \\ Seattle, WA 98195, USA \\ email: slh@astro.washington.edu
}

\begin{abstract}
We are investigating if the orbital geometry of exoplanets affects the activity of their host star by studying a sample of planetary systems known to contain massive planets on short period, highly elliptical orbits. While recent studies in the optical, UV, and X-Ray have shown enhanced chromospheric activity for stars hosting exoplanets with orbital semi-major axes less than 0.1 AU (Krejčova 2012, Shkolnik 2013, Kashyap 2008, Poppenhaeger 2010), it is not yet clear whether this activity is driven by magnetic or tidal interactions. We are probing the dependence of star-planet interactions (SPI) on the orbital geometry of the planetary systems by analyzing chromospheric lines (such as Ca II H \& K) for variability phased with the exoplanet's orbit. We have obtained high resolution spectra of several systems with the McDonald 2.1-m Sandiford echelle spectrograph, ARCES on the APO 3.5-m, and for HIRES on Keck I from the Keck Observatory Archive. We describe our methodology and review how our results will use orbital geometry to deduce how planets may affect the activity of their host stars.
\end{abstract}

Keywords. stars: activity, stars: magnetic fields, planetary systems

\section{Introduction}

The discovery of hot Jupiters (periods $\lesssim 4$ days) led to the search for evidence of various interaction scenarios between these massive planets and their host stars. Recent statistical analyses of data in multiple bands has shown increased chromospheric activity in stars harboring planets closer than 0.1 AU (Krejčova 2012, Shkolnik 2013, Kashyap 2008, Poppenhaeger 2010). Ongoing observational work has produced evidence that in some of these systems the magnetic fields of both bodies may interact. The results of these interactions can be explored in the stellar chromosphere by looking for inversions in the line cores of its activity indicators phased with the planet's orbit.

\section{Overview}

Our echelle observations from APO and McDonald are being paired with data from the Keck Observatory Archive (KOA) to construct time series using two different methods to ensure the consistency and significance of our results. The methods are: 1) Compare variations to a normalized continuum window around each line following the procedures of Shkolnik (2008) and Gurdemir (2012). A mean spectra from all of our datasets is 


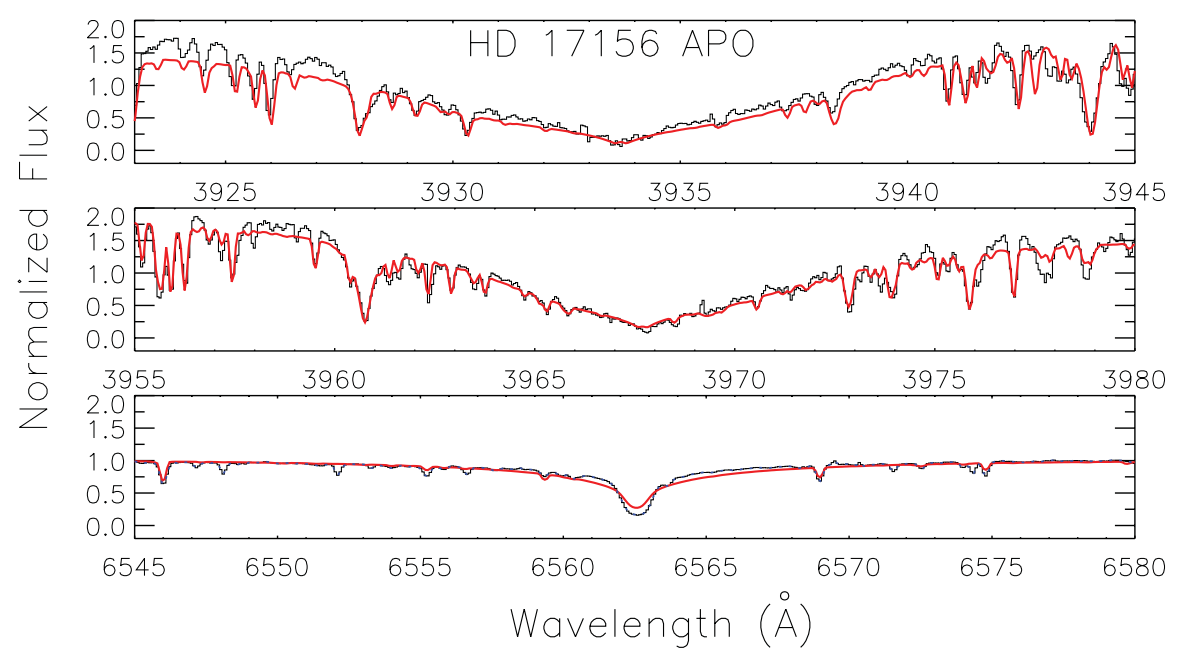

Figure 1. Chromospheric activity indicators from HD 17156 compared to synthetic spectra (in Red) simulated with SME. The top frame shows the total normalized mean flux per wavelength and synthetic spectra for Ca II K, $3934 \AA$, for the 24 Jan 2013 from the APO 3.5m. The center frame is for Ca II H, $3968 \AA$. The bottom frame is for $\mathrm{H} \alpha 6563 \AA$.

then subtracted from the mean nightly spectra. The resultant residuals for each line are then analyzed for evidence of star-planet interactions (SPI). 2) The stellar parameters of each target star are used to construct its synthetic spectrum using the Spectroscopy Made Easy (SME) tool (Valenti \& Piskunov, 1996). This spectrum is subtracted from the normalized mean nightly spectra and the residuals for each line are then analyzed for SPI. An example for the Ca II H\&K and $\mathrm{H} \alpha$ lines, is shown in Figure 1.

We are investigating the planet induced chromospheric activity of each star by examining the Ca II H\&K, Ca II IRT, and $\mathrm{H} \alpha$ lines in our phase observed spectra. To ensure any modulations in the chromospheric lines are not a result of intrinsic stellar activity, we are also monitoring photospheric lines, such as the Al I line (3944 ̊). To differentiate between tidal and magnetic SPI, we are analyzing the constructed time series for modulations phased with the planetary orbit, stellar rotation, and the synodic period of the stellar rotation; in addition to comparing them with various interaction scenarios.

\section{Conclusion}

The full time series for each target system is currently being analyzed and we have good phase coverage of the chromospheric acitivity indicators for several systems that will allow us to draw significant conclusions about the existence of star-planet interactions in planetary systems containing hot-Jupiters with short period, highly eccentric orbits.

\section{References}

Gurdemir, L., Redfield, S., \& Cuntz, M. 2012, PASA, 29, 141

Kashyap, V. L., Drake, J. J., \& Saar, S. H. 2008, ApJ, 687, 1339

Krejčova, T. 2012, $A \& A, 540,82$

Poppenhaeger, K., Robrade, J., \& Schmitt, J. H. 2010, A\&\&A, 515, A98

Shkolnik, E., Bohlender, D. A., Walker, G. A. H., et al. 2008, ApJ, 676, 628

Shkolnik, E. 2013, ApJ, 766, 9S

Valenti J. A., Piskunov N. 1996, A\&AS, 118, 595 